Pacific Journal of Mathematics

ON $\Sigma$-INVERSE SEMIGROUPS 


\section{ON $\Sigma$-INVERSE SEMIGROUPS}

S. SRIBALA

In this paper, the Preston-Vagner theorem on representation of inverse semigroups is extended to a class of uniform inverse semigroups. In this connection the notion of $\Sigma$-uniformity on an inverse simigroup is introduced which is a modification of the congruence uniformity defined by a set of idempotent separating congruences on the inverse semigroup. Such an inverse semigroup is called a $\Sigma$-inverse semigroup. First, it is proved that a $\Sigma$-inverse semigroup is complete if and only if all its maximal subgroups are complete and it is compact if and only if the set of its idempotents is finite and all its maximal subgroups are compact. Next, the symmetric $\Sigma$-inverse semigroup of bi-Lipchitzian maps between $U$-open subsets of an uniform space is defined and finally, it is shown that any $\Sigma$-inverse semigroup can be embedded isomorphically into a symmetric $\Sigma$-inverse semigroup.

1. $\Sigma$-inverse semigroups. We refer to [1] for information on semigroups and to [2] for uniform spaces. We shall always consider symmetric Hausdorff uniformities. Let $(X, \mathfrak{u})$ be a uniform space where $\mathfrak{U}=\left\{U_{k} ; k \in K\right\} . \quad$ A subset $Y$ of $X$ is said to be $U$-open if $x \in Y \Rightarrow$ $U_{k}(x) \subset Y$ for all $k \in K$. A $U$-open subset is both open and closed. The set of all $U$-open subsets of $X$ is closed for the operations of union and intersection and contains the null set $\phi$ and $X$. A mapping $\alpha$ of $X$ into itself is called Lipchitzian if $(x, y) \in U_{k} \Rightarrow(x \alpha, y \alpha) \in U_{k}$. If $\alpha$ is a Lipchitzian map and if $\alpha^{-1}$ exists and is also Lipchitzian, then $\alpha$ is called a bi-Lipchitzian map.

Definition 1. Let $S$ be an inverse semigroup. A symmetric Hausdorff uniformity $\mathfrak{U}=\left\{U_{k} ; k \in K\right\}$ is called a $\Sigma$-uniformity on $S$ if the following conditions hold:

( $\Sigma 1) \quad U_{k} \subseteq \mathscr{H} \quad$ for each $k \in K$.

( $\Sigma 2)$ The maps $\lambda_{a}: x \rightarrow a x$ and $\rho_{a}: x \rightarrow x a$ of $S$ are Lipchitzian maps.

( $\Sigma$ 3) The map $a \rightarrow a^{-1}$ of $S$ is Lipchitzian.

If $\mathfrak{U}$ is a $\Sigma$-uniformity on $S$, then $(S, \mathfrak{u})$ is called a $\Sigma$-inverse semigroup.

In the sequel, $(S, \mathfrak{u})$ denotes a $\Sigma$-inverse semigroup.

Proposition 2. Multiplication in a $\Sigma$-inverse semigroup $(S, \mathfrak{U})$ is uniformly continuous.

Proof. Given $U_{k} \in \mathfrak{U}$ there exists $U_{k_{1}} \in \mathfrak{U}$ such that $U_{k_{1}} \circ U_{k_{1}} \subseteq U_{k}$. 
Then, $\left(x, x^{\prime}\right) \in U_{k_{1}}\left(y, y^{\prime}\right) \in U_{k_{1}} \Rightarrow\left(x y, x^{\prime} y\right) \in U_{k_{1}},\left(x^{\prime} y, x^{\prime} y^{\prime}\right) \in U_{k_{1}}($ by $\Sigma 2) \Rightarrow$ $\left(x y, x^{\prime} y^{\prime}\right) \in U_{k_{1}} \circ U_{k_{1}} \cong U_{k}$.

Proposition 3. The set $E$ of idempotent of $(S, \mathfrak{M})$ is a closed, discrete subset of $S$.

Proof. $E$ is discrete by $\Sigma 1$. If $x \in \bar{E}$, then $U_{k}(x) \cap E \neq \varnothing$ for every $k \in K$. Let $U_{k_{1}} \in \mathfrak{U}$ be such that $U_{k_{1}} \circ U_{k_{1}} \circ U_{k_{1}} \cong U_{k}$. Since $U_{k_{1}}(x) \cap$ $E \neq \varnothing$, there is $e_{k_{1}} \in E$ such that $\left(x, e_{k_{1}}\right) \in U_{k_{1}}$. Then, $\left(x e_{k_{1}}, e_{k_{1}}\right) \in U_{k_{1}}$, $\left(x^{2}, x e_{k_{1}}\right) \in U_{k_{1}}$ by $\Sigma 2$ and so $\left(x^{2}, x\right) \in U_{k_{1}} \circ U_{k_{1}} \cdot U_{k_{1}} \subseteq U_{k}$. This is true for all $k \in K$. Hence $x=x^{2} \in E$ and $E$ is closed.

Proposition 4. Let $T$ be an inverse subsemigroup of a $\Sigma$-inverse semigroup $(S, \mathfrak{n})$. Then $T$ with the relative uniformity is a $\Sigma$-inverse semigroup.

Proof. The relative uniformity for $T$ is given by $\mathfrak{u}_{T}=\left\{U_{k} \cap T \times T\right.$, $k \in K\}$ which satisfies the conditions $\Sigma 2$ and $\Sigma 3$ of Definition 1 . To show that $\Sigma 1$ is satisfied, it is enough to observe that for $a, b \in T$, $a \mathscr{H} b$ in $T$ if and only if $a \mathscr{C} b$ in $S$.

The following can easily be proved.

Proposition 5. The maximal subgroups $H_{e}(e \in E)$ of $S$ are closed.

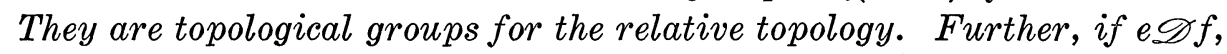
then $H_{e}$ and $H_{f}$ are homeomorphic.

We now give a necessary and sufficient condition for the completeness and compactness of a $\Sigma$-inverse semigroup $(S, \mathfrak{n})$.

THEOREM 6. A $\Sigma$-inverse semigroup $(S, \mathfrak{u})$ is complete if and only if all its maximal subgroups are complete.

Proof. If $S$ is complete, then the subgroup $H_{e}$ are all complete, being closed subsets of $S$. Conversely suppose that each $H_{e}$ is complete. Let $\left\{x_{k} ; k \in K\right\}$ be a Cauchy $K$-net in $S$. Then, given $k \in K$, there exists $k_{1} \in K$ such that $\left(x_{k^{\prime}}, x_{k^{\prime \prime}}\right) \in U_{k}$ for all $k^{\prime}, k^{\prime \prime} \geqq k_{1}$. Hence we can assume without any loss in generality that any given Cauchy $K$-net is contained in a single $\mathscr{H}$ class. Suppose that the Cauchy $K$-net $\left\{x_{k}\right\}$ is contained in the $\mathscr{H}$ class $R_{e} \cap L_{f},(e, f \in E)$. Let $z$ be any element of $R_{e} \cap L_{f}$. Then $\left\{x_{k} z^{-1}\right\}$ is a Cauchy $K$-net in $H_{e}$ and so converges to some point $y \in H_{e}$. Then, we have $\lim _{k \in K} x_{k}=y z$. For, given $U_{k_{0}}$, there is a $k_{1} \in K$ such that $\left(x_{k} z^{-1}, y\right) \in U_{k_{0}}$ for all $k \geqq k_{1}$ and so $\left(x_{k} z^{-1} z, y z\right)=\left(x_{k}, y z\right) \in$ $U_{k_{0}}\left(\right.$ by $\Sigma$ 2) for all $k \geqq k_{1}$ and $\lim _{k \in K} x_{k}=y z$. Thus $S$ is complete. 
THEOREM 7. A $\Sigma$-inverse semigroup $(S, \mathfrak{u})$ is compact if and only if $E$ is finite and each $H_{e}$ is compact.

Proof. If $S$ is compact, it follows that $H_{e}$ is compact and $E$ is finite. Conversely, suppose that $E$ is finite and each $H_{e}$ is compact. We first show that for any $a \in S, H_{a}$ is compact. Let $e, f \in E$ be such that $a \in R_{e} \cap L_{f}$. Then $H_{a}=H_{e} a$ and $x \rightarrow x a$ is uniformly continuous and so $H_{a}$ is compact. The distinct $\mathscr{C}$-classes of $S$ are the nonempty sets $R_{e} \cap L_{f}(e, f \in E)$ and these are only finite in number because $E$ is finite. Thus, $S$ is a union of finite number of compact sets $H_{a}(a \in S)$ and so is compact.

A natural example of a $\Sigma$-uniformity on an inverse semigroup $S$ is given by the congruence uniformity defined by a set $\left\{\theta_{k} ; k \in K\right.$ ) of idempotent separating congruences on $S$ such that given $k_{1}, k_{2} \in K$ there exists $k_{3} \in K$ such that $\theta_{k_{3}} \subset \theta_{k_{1}} \cap \theta_{k_{2}}$ and $\bigcap_{k \in K} \theta_{k}=\tau$, where the uniformity is given by the sets $U_{k}=\left\{(x, y), x, y \in S / x \theta_{k} y\right\}$. In fact, we have

Proposition 8. If $(S, \mathfrak{U})$ is a $\Sigma$-inverse semigroup with idempotent surroundings (i.e., $U_{k} \circ U_{k} \subset U_{k}$ ) then each $U_{k}$ is an idempotent separating congruence on $S$.

2. Symmetric $\Sigma$-inverse semigroup. Let $(X, \mathfrak{u})$ be a uniform space. Let $\mathscr{J}(X)$ be the symmetric inverse semigroup of all partial $(1-1)$ transformations on $X$. Let $\Omega(X)$ be the subset of $\mathscr{J}(X)$ consisting of all partial bi-Lipchitzian maps between $U$-open subsets of $X . \Omega(X)$ is not empty as it contains the null map and identity map of $X$.

Proposition 9. $\Omega(X)$ is an inverse subsemigroup of $\mathscr{J}(X)$.

Proof. If $\alpha \in \Omega(X)$, then $\alpha^{-1}$ also belongs to $\Omega(X)$. Thus it is enough to show that $\Omega(X)$ is a subsemigroup of $\mathscr{F}(X)$. Let $\alpha, \beta \in \Omega(X)$ and

$$
A=\nabla(\alpha) \cap \Delta(\beta) .
$$

(Note: $\Delta(\alpha)$ denotes the domain and $\nabla(\alpha)$ the range of the partial map $\alpha$ ). Then $A$ is $U$-open. If $A=\varnothing$ then $\alpha \beta=0 \in \Omega(X)$. If $A \neq \varnothing$, let $A_{1}=A \alpha^{-1}, A_{2}=A \beta . \quad A_{1}$ is $U$-open, since, $x \in A_{1},(x, y) \in U_{k} \Rightarrow y \in \Delta(\alpha)$, $(x \alpha, y \alpha) \in U_{k} \Rightarrow y \alpha \in U_{k}(x \alpha) \subset A \Rightarrow y \in A_{1}$. Similarly, $A_{2}$ is also $U$-open. It is clear that $\alpha \beta$ is a $(1-1)$ Lipchitzian map of $A$ onto $B$ whose inverse $\beta^{-1} \alpha^{-1}$ is also Lipchitzian. Thus $\alpha \beta \in \Omega(X)$. Hence $\Omega(X)$ is an inverse subsemigroup of $\mathscr{J}(X)$.

The uniformity on $X$ induces in a natural way a uniformity on $\Omega(X)$ which is defined as follows. 
Definition 10. For each $k \in K$ let

$$
\begin{aligned}
U_{k}^{*}= & (\alpha, \beta), \alpha, \beta \in \Omega(X) / \alpha \mathscr{H} \beta,(x \alpha, x \beta) \in U_{k} \\
& \text { for all } \left.x \in \Delta(\alpha),\left(y \alpha^{-1}, y \beta^{-1}\right) \in U_{k} \text { for all } y \in \nabla(\alpha)\right\} .
\end{aligned}
$$

$$
\text { Let } \mathfrak{u}^{*}=\left\{U_{k}^{*} ; k \in K\right\} \text {. }
$$

It is easily verified that $\mathfrak{U}^{*}$ defines a Hausdorff uniformity on $\Omega(X)$ such that $U_{k}^{*} \subset \mathscr{H}$ for all $k \in K$.

Proposition 11. Left and right multiplication in $\Omega(X)$ are Lipchitzian maps.

Proof. Let $\alpha, \beta, \gamma \in \Omega(X),(\alpha, \beta) \in U_{k}^{*}$. Then $\alpha \mathscr{H} \beta,(x \alpha, x \beta) \in U_{k}$ for all $x \in \Delta(\alpha)$ and $\left(y \alpha^{-1}, y \beta^{-1}\right) \in U_{k}$ for all $y \in \nabla(\alpha)$. Let $C=\nabla(\alpha) \cap$ $\Delta(\gamma)=\nabla(\beta) \cap \Delta(\gamma), A_{1}=C \alpha^{-1}, A_{2}=C \beta^{-1}$ and $B=C \gamma$. All these subsets are $U$-open. Now, $x \in A_{1} \Rightarrow x \in \Delta(\alpha) \Rightarrow(x \alpha, x \beta) \in U_{k} \Rightarrow x \beta \in U_{k}(x \alpha) \leqq$ $C \Rightarrow x \in A_{2} \Rightarrow A_{1} \subseteq A_{2}$. Similarly $A_{2} \subseteq A_{1}$ and so $A_{1}=A_{2}$. We have $\alpha \gamma \mathscr{H} \beta \gamma$ since $\Delta(\alpha \gamma)=A_{1}=A_{2}=\Delta(\beta \gamma)$ and $\nabla(\alpha \gamma)=B=\nabla(\beta \gamma)$. Since $\alpha, \beta, \gamma$ are bi-Lipchitzian maps, it follows that $(x \alpha \gamma, x \beta \gamma) \in U_{k}$ for all $x \in \Delta(\alpha \gamma)$ and $\left(y \gamma^{-1} \alpha^{-1}, y \gamma^{-1} \beta^{-1}\right) \in U_{k}$ for all $y \in \nabla(\alpha \gamma)$. Thus $(\alpha \gamma, \beta \gamma) \in U_{k}^{*}$ and multiplication on the right by elements of $\Omega(X)$ is a Lipchitzian map. Similarly, we can show that the left multiplication is also a Lipchitzian map.

Proposition 12. The map $\alpha \rightarrow \alpha^{-1}$ of $\Omega(X)$ is Lipchitzian.

Proof. $(\alpha, \beta) \in U_{k}^{*} \Leftrightarrow \alpha \mathscr{H} \beta,(x \alpha, x \beta) \in U_{k}$ for all $x \in \Delta(\alpha)$ and $\left(y \alpha^{-1}\right.$, $\left.y \beta^{-1}\right) \in U_{k}$ for all $y \in \nabla(\alpha) \Leftrightarrow \alpha^{-1} \mathscr{H} \beta^{-1},\left(y \alpha^{-1}, y \beta^{-1}\right) \in U_{k}$ for all $y \in \Delta\left(\alpha^{-1}\right)=$ $\nabla(\alpha)$ and $(x \alpha, x \beta) \in U_{k}$ for all $x \in \nabla\left(\alpha^{-1}\right)=\Delta(\alpha) \Leftrightarrow\left(\alpha^{-1}, \beta^{-1}\right) \in U_{k}^{*}$.

From the definition of the uniformity $\mathfrak{U}^{*}$ and of Propositions 11 and 12 it follows immediately that $\left(\Omega(X), \mathfrak{U}^{*}\right)$ satisfies the conditions $\Sigma 1-\Sigma 3$ of Definition 1 and thus we have

Theorem 13. $\left(\Omega(X), \mathfrak{U}^{*}\right)$ is a $\Sigma$-inverse semigroup.

Definition $14 . \quad\left(\Omega(X), \mathfrak{U}^{*}\right)$ is called the symmetric $\Sigma$-inverse semigroup of partial bi-Lipchitzian maps on $(X, \mathfrak{u})$ or shortly, the symmetric $\Sigma$-inverse semigroup on $(X, \mathfrak{u})$.

THEOREM 15. Let $(X, \mathfrak{u})$ be a complete uniform space with idempotent surroundings. Then $\left(\Omega(X), \mathfrak{u}^{*}\right)$ is complete.

Proof. Let $\left\{\alpha_{k}, k \in K\right\}$ be a Cauchy $K$-net in $\Omega(X)$. Without loss in generality we can assume that the Cauchy $K$-net is contained in a 
single $\mathscr{H}$-class. Let $A$ be the common domain of $\alpha_{k}, k \in K$ and $B$ their range. We now define a map $\alpha: A \rightarrow B$ as follows. Define $x \alpha=\lim _{k \in K} x \alpha_{k}, x \in A$. Since $B$ is closed and $\left\{x \alpha_{k}\right\}$ is a Cauchy $K$-net in $B$, we have $x \alpha \in B . \quad \alpha$ is a well defined map of $A$ into $B$. Let $x, y \in A$ with $x \alpha=y \alpha$. Then $\lim _{k \in K} x \alpha_{k}=\lim _{k \in K} y \alpha_{k}$. Given $U_{k_{0}}$ we can find $k_{1} \in K$ such that $\left(x \alpha_{k}, x \alpha\right) \in U_{k_{0}},\left(y \alpha_{k}, y \alpha\right) \in U_{k_{0}}$ for all $k \geqq k_{1}$. Thus $\left(x \alpha_{k}, y x_{k}\right) \in U_{k_{0}} \cdot U_{k_{0}}=U_{k_{0}}$ for all $k \geqq k_{1}$. Since $\alpha_{k}^{-1}$ is Lipchitzian, $\left(x \alpha_{k} \alpha_{k}^{-1}, y \alpha_{k} \alpha_{k}^{-1}\right) \in U_{k_{0}}$ for all $k \geqq k_{1}$ i.e., $(x, y) \in U_{k_{0}}$. Since $U_{k_{0}}$ is arbitrary, we have $x=y$ and so $\alpha$ is $(1-1)$. We next show that $\alpha$ is onto. If $y \in B$, then $\left\{y \alpha_{k}^{-1}\right\}$ is a Cauchy $K$-net in $A$ and hence converges to a point $x \in A$. Then $x \alpha=y$. For, given $U_{k_{0}} \in \mathfrak{H}$, there exists $k_{1} \in K$ such that $\left(x, y \alpha_{k}^{-1}\right) \in U_{k_{0}}$ for all $k \geqq k_{1}$ and so $\left(x \alpha_{k}, y\right) \in U_{k_{0}}$ for all $k \geqq k_{1}$. Thus $y=\lim _{k \in K} \cdot x \alpha_{k}=x \alpha$ and $\alpha$ is onto. The maps $\alpha$ and $\alpha^{-1}$ are Lipchitzian. For, let $(x, y) \in U_{k_{0}}, x, y \in A, k_{0} \in K$. Then $\left(x \alpha_{k}, y \alpha_{k}\right) \in U_{k_{0}}$ for all $k \in K$. Since $x \alpha=\lim _{k \in K} x \alpha_{k}, y \alpha=\lim _{k \in K} y \alpha_{k}$ we can find $k_{1} \in K$ such that $\left(x \alpha, x \alpha_{k}\right) \in U_{k_{0}},\left(y \alpha, y \alpha_{k}\right) \in U_{k_{0}}$ for all $k \geqq k_{1}$. Hence $(x \alpha, y \alpha) \in$ $U_{k_{0}} \circ U_{k_{0}} \cdot U_{k_{0}} \subseteq U_{k_{0}}$. Thus $\alpha$ is Lipchitzian. Similarly we can show that $\alpha^{-1}$ is Lipchitzian and so $\alpha \in \Omega(X)$. It now remains only to show that $\alpha=\lim _{k \in K} \alpha_{k}$ in $\Omega(X)$. Since $\left\{\alpha_{k}, k \in K\right\}$ is a Cauchy net, given $U_{k_{0}}^{*}$ we can find $k_{1} \in K$ such that $\left(\alpha_{k}, \alpha_{k^{\prime}}\right) \in U_{k_{0}}^{*}$ for all $k, k^{\prime} \geqq k_{1}$ and so $\left(x \alpha_{k}, x \alpha_{k^{\prime}}\right) \in U_{k_{0}}$ for all $k, k^{\prime} \geqq k_{1}$. Since $x \alpha=\lim _{k \in K} x \alpha_{k}$ we can find $k_{2} \in K$ such that $\left(x \alpha, x \alpha_{k}\right) \in U_{k_{0}}$ for all $k \geqq k_{2}$. Let $k_{3} \in K$ be such that $k_{3} \geqq k_{1}, k_{2}$. Then $\left(x \alpha, x \alpha_{k_{3}}\right) \in U_{k_{0}},\left(x \alpha_{k}, x \alpha_{k_{3}}\right) \in U_{k_{0}},\left(x \alpha, x \alpha_{k_{3}}\right) \in U_{k_{0}}$ for all $k \geqq k_{3}$. Similarly, we can show that if $y \in B$, then $\left(y \alpha^{-1}, y \alpha_{k_{3}}^{-1}\right) \in U_{k_{0}}$, $\left(y \alpha^{-1}, y \alpha_{k}^{-1}\right) \in U_{k_{0}},\left(y \alpha_{k}^{-1}, y \alpha_{k_{3}}^{-1}\right) \in U_{k_{0}}$ for all $k \geqq k_{3}$ and so $\left(\alpha, \alpha_{k}\right) \in U_{k_{0}}^{*}$ for all $k \geqq k_{3}$. Thus $\alpha=\lim _{k \in K} \alpha_{k}$ and so $\Omega(X)$ is complete.

3. Representation of $\Sigma$-inverse semigroups. We now consider the representation of a $\Sigma$-inverse semigroup by partial bi-Lipchitzian maps. Let $(S, \mathfrak{u})$ be a $\Sigma$-inverse semigroup and $\left(\Omega(X), \mathfrak{u}^{*}\right)$ the symmetric $\Sigma$-inverse semigroup on $(S, \mathfrak{u})$. Let $\rho$ be the right regular representation of $S$ in $\mathscr{F}(S)$. We now have

Proposition 16. So is a closed inverse subsemigroup of $\left(\Omega(X), \mathfrak{u}^{*}\right)$.

Proof. The set $S a(a \in S)$ is $U$-open, for, if $x \in S a$ and if $(x, b) \in U_{k}$ for some $k \in K$, then $x \mathscr{H} b$ and $S b=S x \subseteq S a$ and so $b \in S a$. The map $\rho_{a}: S_{a}^{-1} \rightarrow S a$ is $(1-1)$ Lipchitzian between $U$-open sets whose inverse $\rho_{a}^{-1}$ is also Lipchitzian and $\rho_{a} \in \Omega(X)$. Thus $S \rho \subset \Omega(S)$ and $S \rho$ is an inverse subsemigroup of $\Omega(S)$. Now, let $\eta \in \overline{S \rho}$. Then $U_{k}^{*}(\eta) \cap S \rho \neq \phi$ for every $k \in K$. Let $j \in K$ be such that $U_{j} \circ U_{j} \subseteq U_{k}$. Then we can find $a_{j} \in S$ such that $\left(\eta, \rho_{a_{j}}\right) \in U_{j}^{*}$. Then $\Delta(\eta)=S a_{j}^{-1}, \nabla(\eta)=S a_{j}$. Let $e_{j}=a_{j} a_{j}^{-1}$ and $b=e_{j} \eta$. Then $\left(\eta, \rho_{a_{j}}\right) \in U_{j}^{*} \Rightarrow\left(e_{j} \eta, e_{j} \rho_{a_{j}}\right)=\left(b, a_{j}\right) \in U_{j} \Rightarrow$ $b \mathscr{C} a_{j}$. Hence $S b^{-1}=S a_{j}^{-1}=\Delta(\eta)$ and $S b=S a_{j}=\nabla(\eta)$ and so $\eta \mathscr{C} \rho_{b}$. 
Further, if $x \in \Delta(\eta)$, then $\left(x \eta, x a_{j}\right) \in U_{j},\left(x b, x a_{j}\right) \in U_{j}$ and so $(x \eta, x b) \in$ $U_{j} \circ U_{j} \subseteq U_{k}$. This is true for all $k \in K$ and so $x \eta=x b$ for all $x \in \Delta(\eta)$. That is $\eta=\rho_{b}$ and $S \rho$ is closed.

We now have

THEOREM 17. A $\Sigma$-inverse semigroup $(S, \mathfrak{u})$ can be embedded isomorphically in a symmetric $\Sigma$-inverse semigroup.

Proof. The map $\rho: S \rightarrow \Omega(S)$ given by $a \rightarrow \rho_{a}$ is clearly an algebraic isomorphism of $S$ onto $S \rho \subseteq \Omega(S)$. To prove that it is a uniform isomorphism we will show that for $a, b \in S,(a, b) \in U_{k} \Leftrightarrow\left(\rho_{a}, \rho_{b}\right) \in U_{k}^{*}$. Now $(a, b) \in U_{k} \Rightarrow a \mathscr{H} b \Rightarrow \rho_{a} \mathscr{H} \rho_{b}$. If $x \in S a^{-1}, y \in S a$, then $(a, b) \in U_{k} \Rightarrow$ $(x a, x b) \in U_{k}$ and $\left(y a^{-1}, y b^{-1}\right) \in U_{k}$ and thus $\left(\rho_{a}, \rho_{b}\right) \in U_{k}^{*}$. Conversely $\left(\rho_{a}, \rho_{b}\right) \in U_{k}^{*} \Rightarrow S a=S b, S a^{-1}=S b^{-1} \Rightarrow a \mathscr{H} b$. So, if $a, b \in R_{e} \cap L_{f}$, then $(a, b)=\left(e \rho_{a}, e \rho_{b}\right) \in U_{k}$. Thus $\rho$ is a uniform isomorphism of $(S, \mathfrak{u})$ onto a closed $\Sigma$-inverse subsemigroup of $\left(\Omega(S), \mathfrak{U}^{*}\right)$.

\section{REFERENCES}

1. A. H. Clifford and G. B. Preston, Algebraic theory of semigroups Vols. 1 and 2, Amer. Math. Soc., Survey No. 7 (1961); (1967).

2. J. L. Kelley, General Topology, Van Nostrand.

Received August 11, 1972. This paper forms a part of the Ph. D. thesis submitted to the University of Madras in 1969. The author is indebted to Professor V. S. Krishnan who acted as supervisor for his valuable help and encouragement.

The Ramanujan Institute for Advanced Study in Mathematics UNIVERSITY OF MADRAS

MADRAS-5, INDIA. 


\section{PACIFIC JOURNAL OF MATHEMATICS}

\section{EDITORS}

RICHARD ARENS (Managing Editor)

University of California

Los Angeles, California 90024

\section{R. A. Beaumont}

University of Washington

Seattle, Washington 98105

\section{J. DuGundJI*}

Department of Mathematics

University of Southern California

Los Angeles, California 90007

D. Gilbarg and J. Milgram

Stanford University

Stanford, California 94305

ASSOCIATE EDITORS
E. F. BECKENBACH
B. H. NeumanN
F. WOLF
K. YoSHIDA

\section{SUPPORTING INSTITUTIONS}

UNIVERSITY OF BRITISH COLUMBIA

CALIFORNIA INSTITUTE OF TECHNOLOGY

UNIVERSITY OF CALIFORNIA

MONTANA STATE UNIVERSITY

UNIVERSITY OF NEVADA

NEW MEXICO STATE UNIVERSITY

OREGON STATE UNIVERSITY

UNIVERSITY OF OREGON

OSAKA UNIVERSITY

\author{
UNIVERSITY OF SOUTHERN CALIFORNIA \\ STANFORD UNIVERSITY \\ UNIVERSITY OF TOKYO \\ UNIVERSITY OF UTAH \\ WASHINGTON STATE UNIVERSITY \\ UNIVERSITY OF WASHINGTON \\ AMERICAN MATHEMATICAL SOCIETY \\ NAVAL WEAPONS CENTER
}

The Supporting Institutions listed above contribute to the cost of publication of this Journal, but they are not owners or publishers and have no responsibility for its content or policies.

Mathematical papers intended for publication in the Pacific Journal of Mathematics should be in typed form or offset-reproduced, (not dittoed), double spaced with large margins. Underline Greek letters in red, German in green, and script in blue. The first paragraph or two must be capable of being used separately as a synopsis of the entire paper. Items of the bibliography should not be cited there unless absolutely necessary, in which case they must be identified by author and Journal, rather than by item number. Manuscripts, in duplicate if possible, may be sent to any one of the four editors. Please classify according to the scheme of Math. Rev. Index to Vol. 39. All other communications to the editors should be addressed to the managing editor, or Elaine Barth, University of California, Los Angeles, California, 90024.

50 reprints are provided free for each article; additional copies may be obtained at cost in multiples of 50 .

The Pacific Journal of Mathematics is issued monthly as of January 1966. Regular subscription rate: $\$ 48.00$ a year (6 Vols., 12 issues). Special rate: $\$ 24.00$ a year to individual members of supporting institutions.

Subscriptions, orders for back numbers, and changes of address should be sent to Pacific Journal of Mathematics, 103 Highland Boulevard, Berkeley, California, 94708.

\section{PUBLISHED BY PACIFIC JOURNAL OF MATHEMATICS, A NON-PROFIT CORPORATION}

Printed at Kokusai Bunken Insatsusha (International Academic Printing Co., Ltd.), 270, 3-chome Totsuka-cho, Shinjuku-ku, Tokyo 160, Japan.

* C. R. DePrima California Institute of Technology, Pasadena, CA 91109, will replace J. Dugundji until August 1974.

Copyright (C) 1973 by

Pacific Journal of Mathematics

All Rights Reserved 


\section{Pacific Journal of Mathematics}

\section{Vol. 49, No. $2 \quad$ June, 1973}

Wm. R. Allaway, On finding the distribution function for an orthogonal polynomial

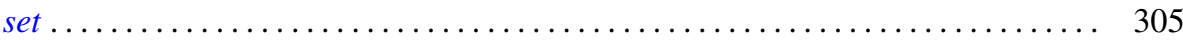

Eric Amar, Sur un théorème de Mooney relatif aux fonctions analytiques bornées... . 311

Robert Morgan Brooks, Analytic structure in the spectrum of a natural system . . . . 315

Bahattin Cengiz, On extremely regular function spaces . . . . . . . . . . . . . . 335

Kwang-nan Chow and Moses Glasner, Atoms on the Royden boundary . . . . . . . . . 339

Paul Frazier Duvall, Jr. and Jim Maxwell, Tame $Z^{2}$-actions on $E^{n} \ldots \ldots \ldots \ldots \ldots . .349$

Allen Roy Freedman, On the additivity theorem for $n$-dimensional asymptotic

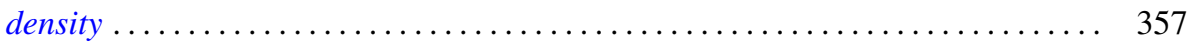

John Griffin and Kelly Denis McKennon, Multipliers and the group $L_{p}$-algebras . . . 365

Charles Lemuel Hagopian, Characterizations of $\lambda$ connected plane continua ....... 371

Jon Craig Helton, Bounds for products of interval functions . . . . . . . . . . . 377

Ikuko Kayashima, On relations between Nörlund and Riesz means . . . . . . . . . . 391

Everett Lee Lady, Slender rings and modules . . . . . . . . . . . . . . . . . . 397

Shozo Matsuura, On the Lu Qi-Keng conjecture and the Bergman representative

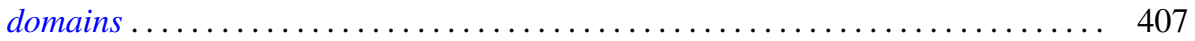

Stephen H. McCleary, The lattice-ordered group of automorphisms of an $\alpha$-set . . . 417

Stephen H. McCleary, o-2-transitive ordered permutation groups .......... 425

Stephen H. McCleary, o-primitive ordered permutation groups. II . . . . . . . . . 431

Richard Rochberg, Almost isometries of Banach spaces and moduli of planar

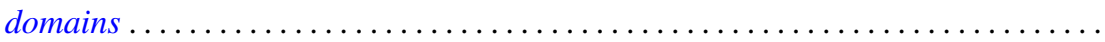

R. F. Rossa, Radical properties involving one-sided ideals . . . . . . . . . . . . . 467

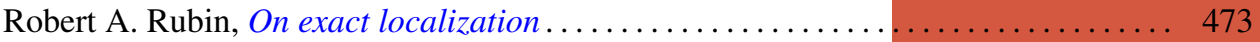

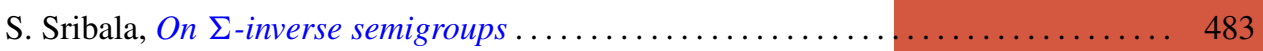

H. M. (Hari Mohan) Srivastava, On the Konhauser sets of biorthogonal polynomials suggested by the Laguerre polynomials ...................... 489

Stuart A. Steinberg, Rings of quotients of rings without nilpotent elements . ...... 493

Daniel Mullane Sunday, The self-equivalences of an $H$-space . . ............ 507

W. J. Thron and Richard Hawks Warren, On the lattice of proximities of $\check{C} e c h$

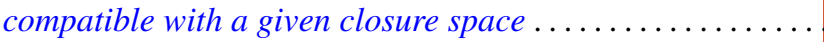

Frank Uhlig, The number of vectors jointly annihilated by two real quadratic forms determines the inertia of matrices in the associated pencil .

Frank Uhlig, On the maximal number of linearly independent real vectors annihilated simultaneously by two real quadratic forms ..............

Frank Uhlig, Definite and semidefinite matrices in a real symmetric matrix pencil . . 561

Arnold Lewis Villone, Self-adjoint extensions of symmetric differential operators . . . 569

Cary Webb, Tensor and direct products . ....................... 579

James Victor Whittaker, On normal subgroups of differentiable

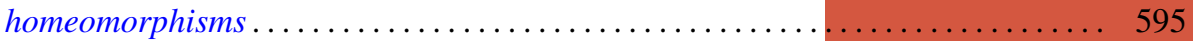

Jerome L. Paul, Addendum to: "Sequences of homeomorphisms which converge to

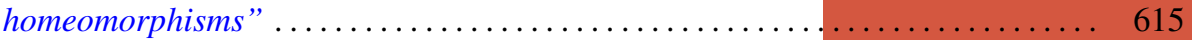

David E. Fields, Correction to: “Dimension theory in power series rings” ........ 616

Peter Michael Curran, Correction to: “Cohomology of finitely presented groups”. . . 617

Billy E. Rhoades, Correction to: “Commutants of some Hausdorff matrices” ...... 617

Charles W. Trigg, Corrections to: "Versum sequences in the binary system” ...... 619 